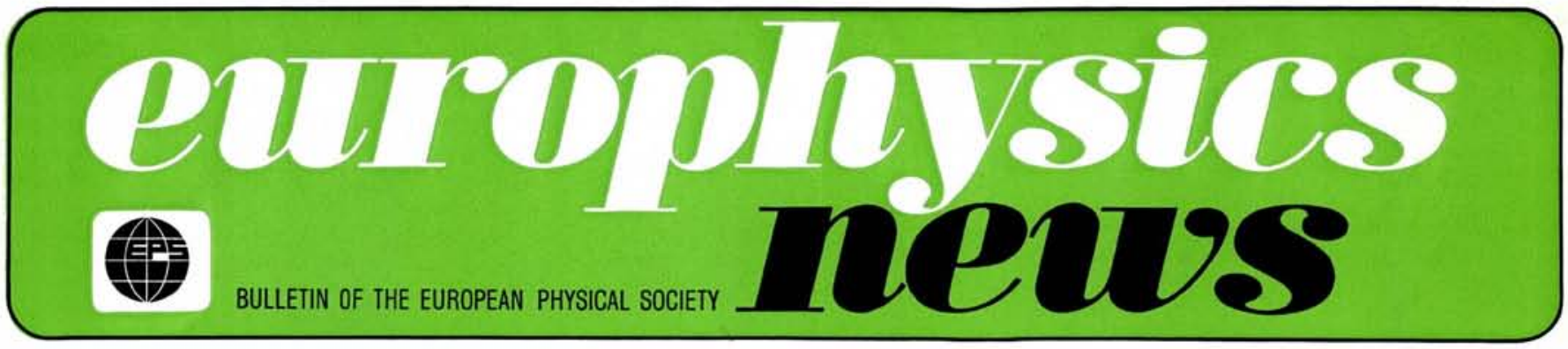

J.A. Volume $8 \quad$ Number 5

May 1977

\title{
DIACETYLENE POLYMERS
}

PRODUCTION AND PROPERTIES OF SINGLE CRYSTALS

D. Bloor, London

(Dept of Physics, Queen Mary College)

Perhaps the most striking property of polymers is their complexity at the molecular level. Biopolymers have complex conformations and arrangements of the chemical sub-units. Synthetic polymers have much simpler chemical structures, but their morphology remains complex. This complexity obscures the intrinsic anisotropy of the polymer chains and results in the physical properties of bulk samples being isotropic. While this is valuable in many technological applications, it has hindered the observation and understanding of the intrinsic molecular properties of polymers.

A proper understanding of polymer morphology has required many years of careful research. Once Staudinger had established that macromolecules made from a linear array of simple repeat units existed, it was assumed that they would form amorphous solids consisting of a spaghetti-like mass of entangled chains. From this, emerged the fringed-micelle model with locally ordered and disordered regions and polymer chains passing through both sorts of region. Finally, after the elegant work of Keller at Bristol, the picture of chain folded lamellar crystals was accepted. Polymer chains are primarily confined to the crystalline lamellae or to the intervening amorphous regions. Bulk samples can be characterized by their degree of crystallinity, which may range from zero to well over $90 \%$. However, since the lamellae are microscopic, it is the bulk morphology which is still the dominant factor in determining physical properties. The difficulty encountered in observing molecular properties is illustrated by the fact that evidence has been produced favouring either, a tight molecular fold at the lamellar surface with adjacent reentry of the molecule or, a loose fold with random re-entry.

With a better understanding of polymer morphology has come the realization that more perfect molecular ordering is possible. Mechanical deformation of polymeric solids and extrusion of melts have produced anisotropic samples in which many of the molecules have considerable chain extension. Such samples, though technologically interesting, are still far from single crystals. The most direct route to such samples is to form the molecular and the crystal structures simultaneously. In the few instances where this has been found to occur, the crystals formed are still microscopic. An alternative method is to initiate polymerization in a monomer crystal and produce a polymer crystal which retains the order of the monomer crystal. This ideal situation has recently been found to obtain for a number of monomers containing diacetylene units.

The interest in the resulting diacetylene polymers stems from the fact that, in addition to providing model systems for studying mechanical properties, they exhibit unusual optical and electrical properties. These originate in the conjugaticn of the diacetylene polymer backbone, as indicated in Fig. 1. Not all the carbon 2p-electrons are built into $\sigma$-bonding states. Some occupy atomic $\mathrm{p}_{\mathrm{z}}$-orbitals, orthogonal to the plane of the backbone, which can overalp to form delocalized $\pi$-states. If the carbon atom separations are equal, the de-localized states form a continuous energy band, which is half filled, i.e. the chain will be metallic. If the carbon separations are unequal. the $\mathrm{p}_{\mathrm{z}}$-electrons are lo- calized in $\pi$-bonds between the closer atoms and a gap forms in the energy band corresponding to the energy required to excite the r-electrons from a localized to a de-localized state. The polymer chain will now be a semiconductor with a large band gap, of the order $2 \mathrm{eV}$. In relation to currently popular quasi-one dimensional metallic systems, diacetylene polymers are systems far below their Peierls transition.

In addition to studies of the fundamental properties of diacetylene polymers, the polymerization process has attracted interest, a fuller understanding of this process being a step towards the molecular engineering of ordered polymers.

As with many phenomena, diacetylene polymerization was observed, but not understood, in the nineteenth century. More recently, the colouring of crystals of natural and synthetic diacetylenes has been described as a polymerization or a decomposition, but treated only as a nuisance. Early X-ray structural studies of diacetylenes reported sample blackening, but polymer formation was discounted as there was no amorphous scattering. It was not until 1969 that these reactions

\section{Contents}

Diacetylene Polymers . . . . 1

Applied Physics - an

Opportunity for EPS .

Luminescence Processes

in Phospors

SPS Inauguration .

gnetic Field.

ESA's Geostationary Satellites 11

Society News . . . . . 12 


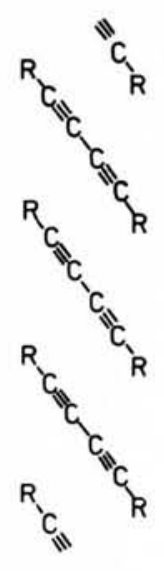

MONOMER

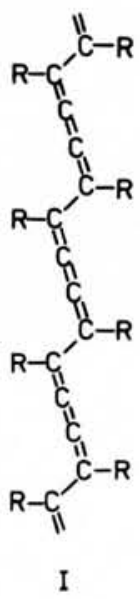<smiles>[R]C(C)=C([R])C=CC([R])=C([R])CCC([R])=C([R])C=CC([R])=C([R])C</smiles>

II

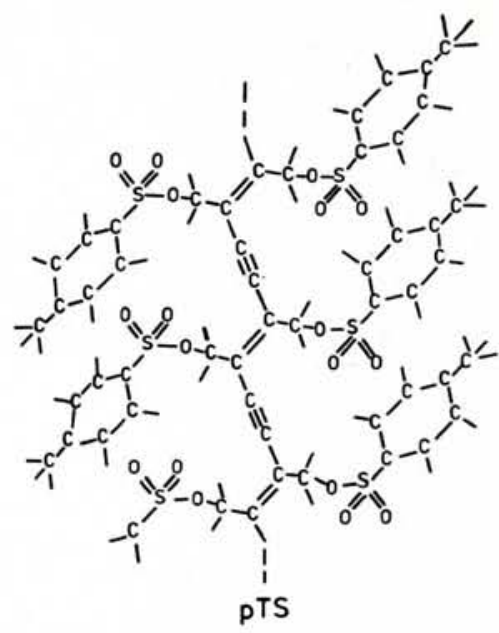

have been observed by electron spin resonance, and identified as ground state triplet radicals. Such carbene radicals have been suggested to provide a mechanism in which the final chain structure is reached by a single step. The full properties of these radicals are still under investigation. The disappearance of these signals in fully polymerized crystals suggests a polymer chain length of $10^{6}$ or more repeat units, i.e. chain lengths of a few tenths of a millimetre. However, the exact chain lengths and distribution have yet to be determined.

Thus, though an elementary understanding of diacetylene polymerization has been obtained, many details remain obscure. This means that while in principle monomer molecules can be "designed" to produce perfect polymer crystals, this is far from being realised in practice. Studies of new materials remain, therefore, a somewhat hit or miss business depending on the skill and intuition of the synthetic chemist.

\section{Physical Properties}

Because of the size and perfection of pTS cyrstals, most studies of the physical properties have been made on this polymer. The optical anisotropy of the polymer crystals is immediately obvious. They have a golden metallic lustre for light polarized parallel to the polymer chains and are black and weakly reflecting for light polarized normal to the chains. Similar anisotropy is observed for other diacetylene polymers but in general their spectra show far less detail than those of $\mathrm{pTS}$.

The spectra of PTS have been extensively studied ${ }^{3}$ ), both by reflection from polymer crystals and by transmission through monomer crystals containing less than $1 \%$ polymer. Thermal polymerization appears to proceed by a chain reaction so that 
partially polymerized samples contain long polymer chains randomly distributed in the monomer matrix, i.e. they are orientated matrix-isolated samples. However, due to the strength of the polymer absorption, care must be taken in the interpretation of transmission to distinguish between effects which arise from the pleochroism of the sample and the intrinsic polymer absorption. Typical reflection and transmission spectra are shown in Fig. 2. An intense absorption, polarized parallel to the polymer chains, occurs at about $2 \mathrm{eV}$ with a series of vibrational side-bands extending into the blue. By comparison with Raman spectra, described below, the sidebands can be identified as polymer backbone stretching vibrations. At low temperatures, the absorptions are split, as shown in Fig. 2. This splitting has not been observed in other diacetylene polymers and is due to a rearrangement of the toluene sulphonate side-groups at about $200 \mathrm{~K}$. This rearrangement occurs in both polymer and monomer crystals producing two non-equivalent polymer chains. The splitting appears, therefore, to be due to mechanical deformation of the polymer backbone though an alternative mechanism, involving interaction of pairs of polymer chains, has been suggested.

The polymer chain absorption is very strong, the maximum absorption coefficient is about $10^{5} \mathrm{~cm}^{-1}$, and anisotropic; the absorption tensor has principal components in the ratio of $1.5 \times 10^{-2}: 10^{-5}$. The absorption line has an asymmetric profile with a sharp rising edge and a slowly falling high energy tail. The absorption tail fits the shape of a one-dimensional van Hove singularity for pTS and a number of other diacetylene polymers. These results are consistent with the interpretation of the absorption as an interband transition of the $\pi$-electron system. However, asymmetric Lorentzian lineshapes are observed for excitons with intermediate phonon coupling and an alternative model is a Wannier exciton, which lies just below the conduction band edge and steals intensity from the inter-band transition. The absorption data, on their own, are insufficient to distinguish between these two possibilities.

The intense electronic absorption leads to a dramatic resonant enhancement of the Raman scattering of vibrations which couple with the electron system. Since the $\pi$-electrons are localized in the polymer backbone, skeletal vibrations dominate the Raman spectrum. This is illustrated in Fig. 3 which shows the Raman spec- trum of pTS at two different excitation wavelengths. The unit cell contains two repeat units, with the structure shown in Fig. 1, but only four vibrations, the stretching modes of the backbone, appear strongly in the resonantly enhanced spectrum. At the shorter excitation wavelength, harmonic and combination frequencies appear, an effect characteristic of resonant Raman scattering. Since the vibrational frequencies are sensitive to small changes in force constants, they reveal fine details of the backbone structure. For example, in the low temperature phase of pTS, some vibrations are observed to split. By tuning the Raman source, a dye laser. through the absorption peaks, one member of each split vibration was shown to be coupled to one of the electronic absorption peaks and not the other.

The resonant enhancement of the backbone vibrations closely follows the absorption profile, behaviour which has been observed in resonant scattering involving excitons. The spectra show an unusual intensity distribution, the double-bond vibration near $1500 \mathrm{~cm}^{-1}$ is more intense than the triple-bond vibration near $2100 \mathrm{~cm}^{-1}$, see Fig. 3. In general the reverse is true since triple bonds are more polarizable than double bonds. This, and the predominance of combinations involving the double-bond vibration, indicate that electronic ex-
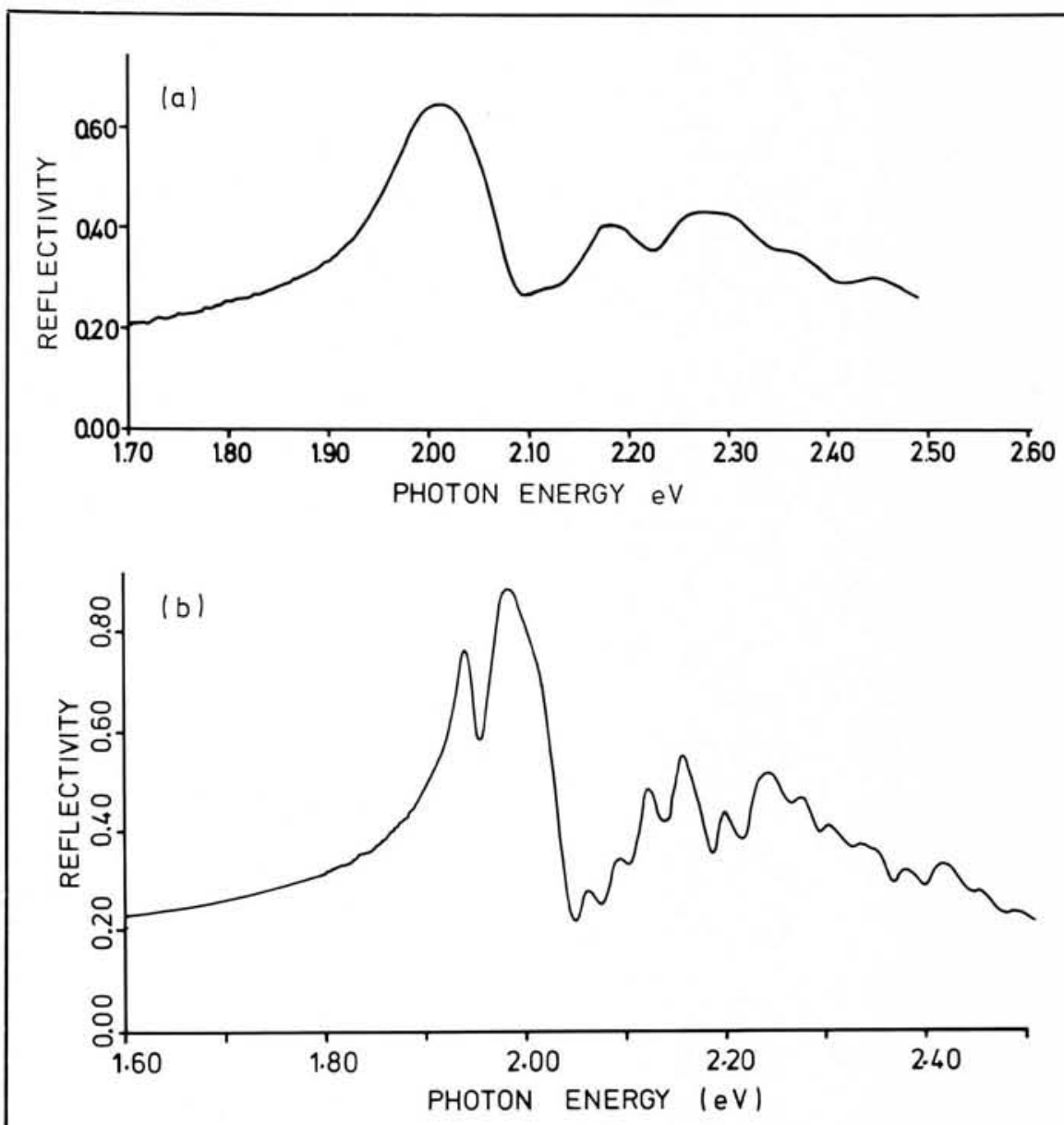

Fig. 2 TS polymer spectra (a) polymer reflection at 300 $K$, (b) polymer reflexion at $4.2 K$ and (c) transmission of a monomer crystal containing less than $1 \%$ polymer at 4.2 K. All spectra were measured with the light polarized parallel to the polymer chains.

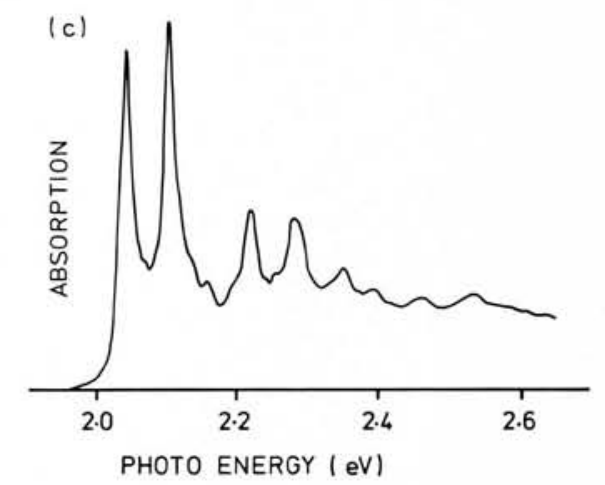


citation has a large electron density in the vicinity of the double bond. These results suggest that the $2 \mathrm{eV}$ absorption is due to an exciton.

Measurements of electrical conductivity provide another probe to test the nature of the electronic states of the polymer backbone ${ }^{4}$ ). pTS is in principle a semi-conductor, but the large energy gap, $2 \mathrm{eV}$ or more, makes it a good insulator with typical dark conductivities of the order of $10^{-12}$ mho. Higher photoconductivity is expected, but in practice, the much smaller penetration depth for light at the absorption peak, leads to a decrease in the measured photocurrent. When allowance is made for this effect, a photoconduction edge is observed at $2 \mathrm{eV}$ with current parallel to the polymer chains three orders of magnitude greater than that perpendicular. The quantum efficiencies and carrier mobilities deduced from these results are dependent on the model used. If space charge current limitation is considered important, the quantum efficiency is very low and the mobility is high, suggesting interband transitions with strong geminate recombination of the carrier pairs. If space charge is considered negligible, the quantum efficiency is found to be higher and the mobility lower, as expected for auto-ionization of excitons. Thus, until the role of space charge is fully understood, these measurements cannot identify unambiguously which model for the $2 \mathrm{eV}$ electronic excitation is correct.

Conjugated polymers have been observed to exhibit paramagnetism. A singlet electron spin resonance spectrum with no hyperfine structure and a wide range of spin concentrations has been observed in many different materials and numerous models for the paramagnetic species have been proposed. pTS has been used as a model system to investigate this paramagnetism ${ }^{5}$ ). A weak singlet spectrum is observed with a strength dependent on sample perfection. The spin concentration is least in the most perfect samples and the paramagnetism has been identified as a defect property. However, since chains completely free of defects cannot be obtained, some paramagnetism will be present in all samples.

More conventional polymer properties, dielectric, thermal and mechanical, have been less thoroughly studied. Negative thermal expansion has been observed in the chain direction and attributed to chain contraction during torsional motion. However, accurate studies of pTS show a small but positive expansion coefficient at

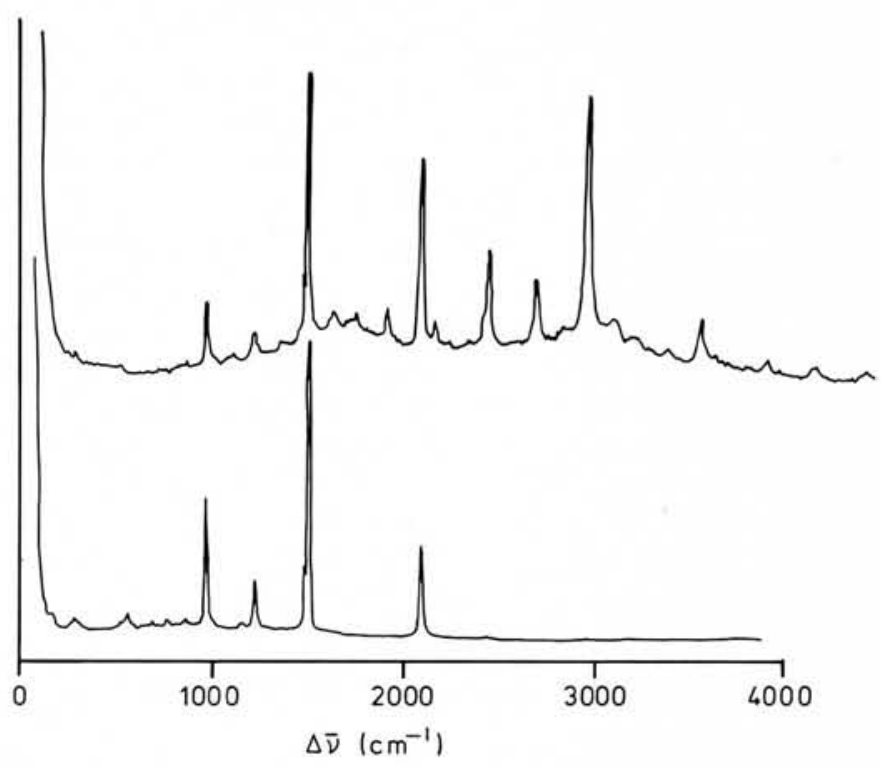

Fig. 3 Raman spectra of pTS recorded for excitation at $647.1 \mathrm{~nm}$ wavelength, lower curve, and $562.2 \mathrm{~nm}$ wavelength, upper curve, at room temperature. Single, double and triple bond stretching vibrations occcur near $1000 \mathrm{~cm}^{-1}, 1500 \mathrm{~cm}^{-1}$ and $2100 \mathrm{~cm}^{-1}$ respectively.

room temperature. The mechanical properties are anisotropic but the measured elastic modulus and ultimate strength are lower than those expected for isolated chains. The modulus is reduced by the large crosssectional area per chain due to the presence of the large side-groups which do not contribute significantly to the elasticity. The ultimate strength is reduced by failure at surface and bulk defects. pTS crystals have distinct metal-like twin features with the twin planes normal to the polymer chains. These twins differ from those reported in microscopic polymer crystals where the twin planes are parallel to the chain direction. Despite the fact that PTS crystals cleave easily they can, like metals, be rolled out to form thin sheets. Such behaviour is of considerable interest to materials scientists and there is scope for much more work in this area in the future.

\section{Future Developments}

We have seen that there is still much room for improvement in our understanding of diacetylene polymers. Whatever the nature of the $2 \mathrm{eV}$ excited electronic state of the polymer backbone, there remains the possibility of energy transport along the polymer chains. This offers potential technological applications and interesting parallels with energy transport in living sytems and simple molecular crystals.

A more quantitative understanding of the parameters necessary for the production of good polymer crystals is needed. This would pave the way to genuine, rather than speculative, molecular engineering of polymer crystals designed to enhance particular properties. At the simplest level this would involve a reduction in sidegroup size to obtain better mechanical properties. More challenging is the modification of chain properties by interacting side-groups. Little's model excitonic high temperature superconductor could be realized by a diacetylene chain with polarizable side-groups. Alternatively the diacetylene chains could be used as frameworks to stack sidegroups in a well defined array, constrained TCNQ stacks could have very interesting properties. Finally it should be noted that diacetylene polymers are the most nearly one-dimensional of the quasi one-dimensional materials currently under investigation. They are, therefore, excellent model materials for the exploration of the physics of one-dimension.

\section{References}

1. WEGNER G., Z. Naturf. 24b (1969) 824 and Chimia (Zürich) 28 (1974) 475.

2. BAUGhMAN R. H., J. Polym. Sci., Polym. Phys. Ed. 12 (1974) 1511.

3. BLOOR D. and PRESTON F. H., Phys. Stat. Sol. (a) 37 (1976) 427 ; 39 (1977) 607 and 40 (1977) and earlier references quoted therein.

4. LOCHNER K., REIMER B. and BÄSSLER., Phys. Stat Sol. (b) 76 (1976) 533 ; Chem. Phys. Lett. 41 (1976) 388 and earlier references quoted therein.

5. BLOOR D., Magnetic Resonance and Related Phenomena (Ed. H. Brunner, K.H. Hauser and D. Schweitzer, Groupement Ampere, Geneva), 1976, p. 47. 